\title{
sp-hybridized carbon allotrope molecular structures: An ongoing challenge for density-functional approximations
}

Cite as: J. Chem. Phys. 151, 211104 (2019); https://doi.org/10.1063/1.5133639

Submitted: 25 October 2019 . Accepted: 13 November 2019 . Published Online: 04 December 2019

Éric Brémond (D), Ángel José Pérez-Jiménez (D), Carlo Adamo (D), and Juan Carlos Sancho-García (iD)
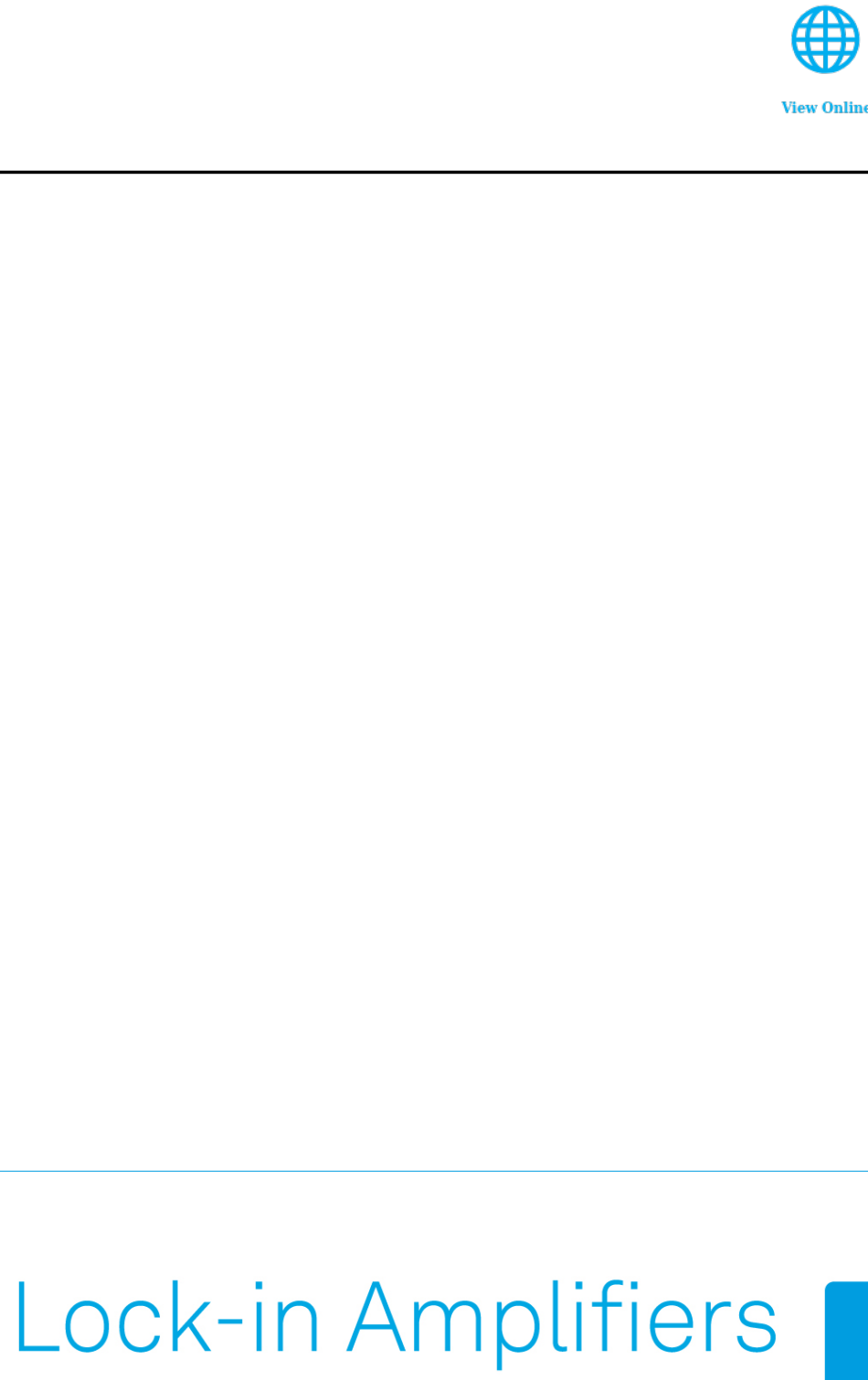

... and more, from DC to $600 \mathrm{MHz}$ Watch 


\title{
sp-hybridized carbon allotrope molecular structures: An ongoing challenge for density-functional approximations
}

\author{
Cite as: J. Chem. Phys. 151, 211104 (2019); doi: 10.1063/1.5133639 \\ Submitted: 25 October 2019 • Accepted: 13 November 2019 • \\ Published Online: 4 December 2019
}

\author{
Éric Brémond, ${ }^{1, a)}$ (D) Ángel José Pérez-Jiménez, ${ }^{2}$ (iD Carlo Adamo

\begin{abstract}
AFFILIATIONS
${ }^{1}$ Université de Paris, ITODYS, UMR CNRS 7086, 15 Rue J.-A. de Baiff, F-75013 Paris, France

${ }^{2}$ Departamento de Química Física, Universidad de Alicante, E-03080 Alicante, Spain

${ }^{3}$ Chimie ParisTech, PSL Research University, CNRS, Institute of Chemistry for Life and Health Sciences (i-CLeHS), FRE 2027, F-75005 Paris, France

${ }^{4}$ Institut Universitaire de France, 103 Boulevard Saint Michel, F-75005 Paris, France
\end{abstract}

a)Electronic addresses: eric.bremond@u-paris.fr and jc.sancho@ua.es

\begin{abstract}
The recent synthesis of $\mathrm{C}_{18}$ monocyclic ring constitutes a major breakthrough as a new all-carbon disclosed form. However, modern density functional theory approaches do not lead to the correct experimental polyynic structure and favor the cumulenic one instead. We demonstrate here that this serious drawback can be solved by recently developed range-separated nonempirical schemes, independently of which kind of functional is being applied (i.e., semilocal, hybrid, or double-hybrid).
\end{abstract}

Published under license by AIP Publishing. https://doi.org/10.1063/1.5133639

Chemistry is still defeating matter to create newly designed molecular forms. Very recently, synthetic chemists made the firstever ring exclusively composed of carbon atoms, ${ }^{1}$ which constitutes "a new form of matter" according to the accompanying generalpublic news, ${ }^{2}$ thus illustrating the hopes and efforts behind this longstanding achievement. ${ }^{3,4}$ This cyclo[18]carbon $\left(\mathrm{C}_{18}\right)$ molecule was isolated, and its structure was characterized by high-resolution atomic spectroscopy, ${ }^{5,6}$ revealing a nanoring (see Fig. 1) with alternating single and triple bonds (i.e., a polyynic structure) and not made entirely of double bonds (i.e., a cumulenic structure). Unfortunately, the triple and single bond lengths could not be measured experimentally at this stage.

Whereas the simplest $a b$ initio (and uncorrelated) HartreeFock (HF) method correctly predicted the polyynic form as the most stable one, early higher-level theoretical predictions for cyclic carbon compounds $C_{n}$ led to completely different results depending on the theoretical method selected, ${ }^{7}$ also influenced by the use of moderate size basis sets (e.g., 6-31G ${ }^{*}$ ) and the vast variety of existing combinations. On the other hand, these linear or cyclic carbon-made $\mathrm{C}_{\mathrm{n}}$ systems are known to historically be particularly challenging for the (widely applied) Density Functional Theory (DFT) due to the delocalization and/or many-electron self-interaction error. ${ }^{8,9}$ Therefore, depending on the functional chosen, the artificial stabilization of delocalized structures could mask any intended prediction in real systems, ${ }^{10}$ thus stressing the need to cure this error for state-of-theart computations. ${ }^{11}$ Actually, most of the studies reported so far used the common PBE, BLYP, PBE0, or B3LYP functionals, with cc-pVTZ or larger basis sets, and wrongly predicted the cumulenic structure as the global energy minimum with a $\mathrm{R}(\mathrm{C}=\mathrm{C})$ distance around 1.27-1.29 $\AA^{1}$. $^{12}$

Considering this challenging issue, not fully solved yet, and given the large amount of density functionals available in common codes, the newly synthesized $\mathrm{C}_{18}$ allotrope becomes an excellent benchmark to assess last-generation density functional approximations providing the right answer by the right reason at a moderate computational cost. For this purpose, we will systematically apply here a set of PBE-based parameter-free (i.e., minimally empirical) exchange-correlation functionals, together with the sufficiently large def2-TZVP basis set, to optimize the structure of both cumulenic and polyynic forms. Note that we also systematically climb step by 


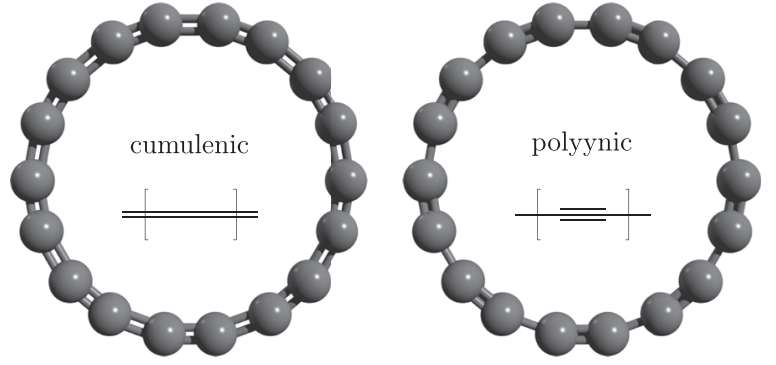

FIG. 1. Cumulenic (left) and polyynic (right) forms of $C_{18}$ nanoring with the inset showing the corresponding Lewis structure.

step the hierarchy of DFT methods, in search of the highest accuracy along a more complex functional form. We did not impose higher than $\mathrm{C}_{s}$ and $\mathrm{C}_{1}$ symmetry groups to the cumulenic and polyynic structures, respectively, to avoid any undesired influence of symmetry breaking and/or stabilization effects upon reducing the $\mathrm{D}_{18 \mathrm{~h}}$ (cumulenic) or $\mathrm{D}_{9 \mathrm{~h}}$ (polyynic) symmetries. To further confirm these results and to verify that they do not depend on the addition of diffuse functions and/or the use of other family of basis sets, we also employed the aug-cc-pVTZ one without any meaningful impact. The nature of a minimum was confirmed in all cases by the presence of $3 \mathrm{~N}-6$ real frequencies, and the optimizations did not lead to any flattening or out-of-plane distortion (i.e., all bond angles having a value of $160^{\circ}$ ). We performed all the computations with the Gaussian 16 package (Revision B.01) using a tight threshold as a convergence criteria in addition to an ultrafine integration grid.

Additionally, we would like to remark that (i) going from semilocal (PBE) to hybrid (PBE0) and double-hybrid (PBE0-DH) functionals allows us to pioneeringly explore the dependence of the results on the functional form, including the modern double-hybrid expressions and (ii) using different hybrid (e.g., PBE0 and PBE0-1/3) and double-hybrid (e.g., PBE0-DH vs PBE-QIDH) expressions also allows us to disentangle the effect (if any) of their composition on the final results. ${ }^{14}$ For a review on double-hybrid density functionals, see Ref. 15. Table I summarizes all the functionals selected, according to the different weights given to their entering ingredients from the general expression

$$
\begin{aligned}
E_{x c}[\rho]= & \lambda_{x} E_{x}^{E X X}[\phi]+\left(1-\lambda_{x}\right) E_{x}[\rho]+\lambda_{c} E_{c}^{P T 2}\left[\phi, \phi^{\prime}\right] \\
& +\left(1-\lambda_{c}\right) E_{c}[\rho],
\end{aligned}
$$

TABLE I. Detailed values for the exchange-correlation functionals used in this study: $\lambda_{x}$ and $\lambda_{c}$ define the functional form [see Eq. (1)], and $\mu\left(\right.$ bohr $\left.^{-1}\right)$ is the range-separation value [see Eq. (2)].

\begin{tabular}{lccc}
\hline \hline Functional & $\mu$ & $\lambda_{x}$ & $\lambda_{c}$ \\
\hline (RSX-)PBE & 0.45 & $\ldots$ & $\ldots$ \\
(RSX-)PBE0 & 0.39 & $1 / 4$ & $\ldots$ \\
(RSX-)PBE0-1/3 & 0.37 & $1 / 3$ & $\ldots$ \\
(RSX-)PBE0-DH & 0.33 & $1 / 2$ & $1 / 8$ \\
(RSX-)PBE-QIDH & 0.27 & $3^{-1 / 3}$ & $1 / 3$ \\
\hline \hline
\end{tabular}

where $E_{x}[\rho]$ and $E_{c}[\rho]$ represent the exchange and correlation energy density approximations, respectively, and $E_{x}{ }^{E X X}[\phi]$ and $E_{c}{ }^{P T 2}[\phi$, $\left.\phi^{\prime}\right]$ are the occupied $(\phi)$ and virtual $\left(\phi^{\prime}\right)$ orbital-dependent exactexchange and 2 nd order perturbative correlation energies, respectively, weighted by the $\lambda_{x}$ and $\lambda_{c}$ values. Note that "RSX" are the corresponding "range-separated exchange" expressions for each of the selected functionals, with $\mu$ being the range-separation $\left(\right.$ bohr $^{-1}$ ) threshold switching the exchange operator $r_{12}{ }^{-1}$ from short- to long-range regimes,

$$
\frac{1}{r_{12}}=\frac{1-\left[\lambda_{x}+\left(1-\lambda_{x}\right) \operatorname{erf}\left(\mu r_{12}\right)\right]}{r_{12}}+\frac{\lambda_{x}+\left(1-\lambda_{x}\right) \operatorname{erf}\left(\mu r_{12}\right)}{r_{12}} .
$$

Note also that those values of $\mu$ exposed in Table I were recently disclosed by the authors of the present work after imposing the recovery of the exact energy of the $\mathrm{H}$ atom, ${ }^{16}$ a physical constraint aiming to reduce the self-interaction error, instead of being considered as a tunable parameter as it is often done in the literature. ${ }^{17}$ This computational protocol will thus allow us to unambiguously confirm if the parameter-free range-separation scheme can lead to accurate results with independence of the functional form selected.

We also concomitantly explored first the application of finitetemperature $\mathrm{DFT}^{18}$ to both cumulenic and polyynic forms, confirming a predominant weak static correlation effects for both structures, which did not prompt us to use multiconfigurational (MC) methods to tackle this challenging issue. ${ }^{19}$ Note also that any active space would need to size-consistently include both $\sigma$ and $\pi$ electrons, with the related computational cost scaling exponentially and/or difficulty in applying these MC methods to other larger systems, as well as their geometry optimization. Furthermore, we have also checked the use of a dispersion-corrected method, ${ }^{20}$ e.g., PBE-D3(BJ), which did not bring any difference with respect to the uncorrected PBE method and still wrongly predicted (vide infra) a cumulene-type global minimum structure, thus showing the negligible influence of intramolecular noncovalent interactions in this case.

Table II gathers the results obtained for both forms of the all-carbon monocyclic ring tackled here at all levels of theory,

TABLE II. Distances (in $\AA$ ) of the global minimum of $\mathrm{C}_{18}$ obtained at different theoretical levels.

\begin{tabular}{lccc}
\hline \hline & & \multicolumn{2}{c}{ Polyynic } \\
\cline { 3 - 4 } Functional & $\mathrm{R}(\mathrm{C}=\mathrm{C})$ & $\mathrm{R}(\mathrm{C} \equiv \mathrm{C})$ & $\mathrm{R}(\mathrm{C}-\mathrm{C})$ \\
\hline PBE & 1.286 & $\ldots$ & $\ldots$ \\
PBE0 & 1.276 & $\ldots$ & $\ldots$ \\
PBE0-1/3 & 1.273 & $\ldots$ & \\
PBE0-DH & 1.274 & $\ldots$ & $\ldots$ \\
PBE-QIDH & 1.277 & $\ldots$ & $\ldots$ \\
RSX-PBE & $\ldots$ & 1.203 & 1.359 \\
RSX-PBE0 & $\ldots$ & 1.201 & 1.357 \\
RSX-PBE0-1/3 & $\ldots$ & 1.200 & 1.356 \\
RSX-PBE0-DH & $\ldots$ & 1.207 & 1.351 \\
RSX-PBE-QIDH & $\ldots$ & 1.220 & 1.341 \\
\hline \hline
\end{tabular}


and this is the main result of the present study, from the pristine (PBE, PBE0, PBE0-1/3, PBE0-DH, and PBE-QIDH) to the range-separated functionals (RSX-PBE, RSX-PBE0, RSX-PBE0-1/3, RSX-PBE0-DH, and RSX-PBE-QIDH). As we can easily see, all the PBE-based functionals predict a cumulenic structure as the global minimum, independently of their functional form, while the RSX-based versions do the opposite in agreement with experiments. The amount of exact-exchange (HF-like) introduced into the hybrid and double-hybrid methods is not enough to provide the correct structure; note that $\lambda_{x}$ spans from $1 / 3$ to $3^{-1 / 3}$ from PBE0 to PBE-QIDH, neither the use of a nonlocal perturbative correction $\left(\lambda_{c}\right)$, in the case of $\mathrm{PBE} 0-\mathrm{DH}$ or $\mathrm{PBE}-\mathrm{QIDH}$, to attain the correct polyynic structure. This is probably due to the fact that MP2/def2-TZVP also led to the wrong cumulenic structure (1.298 $\AA$ ), while HF/def2-TZVP converged on the experimentally observed polyynic form (1.191/1.375 $\AA$ ). We clearly conclude that the use of a range-separation scheme, independently of the underlying functional and reducing considerably the self-interaction error, is key to success.

Note that the excellent results obtained by any of the RSXbased functionals do not rely on the ad hoc tuning of the $\mu$ parameter, neither on an extensive parameterization of the underlying exchange-correlation functional, and keep consistency at all the functional levels, with $\mathrm{R}(\mathrm{C} \equiv \mathrm{C})$ and $\mathrm{R}(\mathrm{C}-\mathrm{C})$ bond lengths comprised in the range 1.20-1.22 $\AA$ and 1.34-1.36 $\AA$, respectively, compared with CCSD/cc-pVDZ calculations for which distances of 1.234 and $1.383 \AA$ A were obtained in the past. ${ }^{21}$ Other highly accurate fixednode diffusion Monte Carlo results, arguably introducing up to $95 \%$ of total correlation energy, also converged to the cyclic polyynic structure, ${ }^{22}$ which is also expected to be the favored form with respect to other $\mathrm{C}_{18}$ isomers such as cages, chains, or graphiticlike structures, ${ }^{23}$ or in the case of Peierls distortions for intermediate size $\mathrm{C}_{4 \mathrm{n}+2}$ carbon chains. ${ }^{24}$ The success of range-separated approximations for this system resembles the previous success of such functionals in describing bond length alternation (BLA) in polyene/polyyne chains $^{25}$ and the improvement obtained when self-interaction corrections are also imposed. ${ }^{26} \mathrm{We}$ also note on passing that in the meanwhile, a paper addressing these systems has recently been published.

In summary, the present work reports how modern rangeseparated schemes can cope with the difficulties of density functional expressions to correctly describe the structural differences between cumulenic and polyynic geometries, which is a paradigmatic example of the competition between localized and delocalized electronic structures. Since experimental findings are the final judges of any discovery, despite previous success with $a b$ initio methods, these RSX-corrected functionals are able to reconcile both worlds, independently of the particular form of the exchange-correlation functional finally selected, be it semilocal, hybrid, or double-hybrid. Despite the interplay of different electronic effects, it seems that exchange contributions are key to obtain the correct structural form, with RSX-corrected functionals providing the right answer at a reasonable computational cost.

E.B. thanks ANR (Agence Nationale de la Recherche) and CGI (Commissariat à l'Investissement d'Avenir) for their financial support for this work through Labex SEAM (Science and Engineering for Advanced Materials and Devices), Grant No. ANR10-LABX-096/ANR-18-IDEX-0001. J.C.S.-G. and A.J.P.-J. thank the Generalitat Valenciana for financial support through Project No. AICO/2018/175. The authors acknowledge the GENCI-CINES for HPC resources (Project Nos. AP010810360 and A0040810359).

\section{REFERENCES}

${ }^{1}$ K. Kaiser, L. M. Scriven, F. Schulz, P. Gawel, L. Gross, and H. L. Anderson, Science 365, 1299-1301 (2019).

${ }^{2}$ D. Castelvecchi, Nature 572, 426 (2019).

${ }^{3}$ G. A. Adamson and C. W. Rees, J. Chem. Soc., Perkin Trans. 1, 1535-1543 (1996). ${ }^{4}$ F. Diederich, Y. Rubin, C. B. Knobler, R. L. Whetten, K. E. Schriver, K. N. Houk, and Y. Li, Science 245, 1088-1090 (1989).

${ }^{\mathbf{5}}$ B. Schuler, S. Fatayer, F. Mohn, N. Moll, N. Pavliček, G. Meyer, D. Peña, and L. Gross, Nat. Chem. 8, 220-224 (2016).

${ }^{6}$ N. Pavliček, P. Gawel, D. R. Kohn, Z. Majzik, Y. Xiong, G. Meyer, H. L. Anderson, and L. Gross, Nat. Chem. 10, 853-858 (2019).

${ }^{7}$ V. Parasuk, J. Almlof, and M. W. Feyereisen, J. Am. Chem. Soc. 113, 1049-1050 (1991).

${ }^{8}$ P. Mori-Sánchez, A. J. Cohen, and W. Yang, J. Chem. Phys. 125, 201102 (2006).

${ }^{9}$ T. Heaton-Burgess and W. Yang, J. Chem. Phys. 132, 234113 (2010).

${ }^{10}$ M. Lundberg and P. E. M. Siegbahn, J. Chem. Phys. 122, 224103 (2005).

${ }^{11}$ X. Zheng, M. Liu, E. Johnson, J. Contreras-García, and W. Yang, J. Chem. Phys. 137, 214106 (2012).

${ }^{12}$ D. A. Plattner and K. N. Houk, J. Am. Chem. Soc. 117, 4405-4406 (1995).

${ }^{13}$ R. O. Jones, J. Chem. Phys. 110, 5189-5200 (1999).

${ }^{14}$ É. Brémond, M. Savarese, A. J. Pérez-Jiménez, J. C. Sancho-García, and C. Adamo, J. Phys. Chem. Lett. 6, 3540-3545 (2015).

${ }^{15}$ É. Brémond, I. Ciofini, J. C. Sancho-García, and C. Adamo, Acc. Chem. Res. 49, 1503-1513 (2016).

${ }^{16}$ É. Brémond, Á. J. Pérez-Jiménez, J. C. Sancho-García, and C. Adamo, J. Chem. Phys. 150, 201102 (2019).

${ }^{17}$ J.-D. Chai and M. Head-Gordon, J. Chem. Phys. 128, 084106 (2008).

${ }^{18}$ S. Grimme and A. Hansen, Angew. Chem., Int. Ed. 54, 12308-12313 (2015).

${ }^{19}$ S. Sen, P. Seal, and S. Chakrabarti, Phys. Rev. B 73, 245401 (2006).

${ }^{20} \mathrm{~S}$. Grimme, A. Hansen, J. G. Brandenburg, and C. Bannwarth, Chem. Rev. 116, 5105-5154 (2016).

${ }^{21}$ S. Arulmozhiraja and T. Ohno, J. Chem. Phys. 128, 114301 (2008).

${ }^{22}$ T. Torelli and L. Mitas, Phys. Rev. Lett. 85, 1702 (2000).

${ }^{23}$ T. W. Yen and S. K. Lai, J. Chem. Phys. 142, 084313 (2015).

${ }^{24}$ C. Neiss, E. Trushin, and A. Görling, ChemPhysChem 15, 2497-2502 (2014).

${ }^{25}$ M. J. G. Peach, E. I. Tellgren, P. Sałek, T. Helgaker, and D. J. Tozer, J. Phys. Chem. A 111, 11930-11935 (2007).

${ }^{26}$ I. Ciofini, C. Adamo, and H. Chermette, J. Chem. Phys. 123, 121102 (2005).

${ }^{27}$ G. B. Baryshnikov, R. R. Valiev, A. V. Kuklin, D. Sundholm, and H. Ågren, J. Phys. Chem. Lett. 10, 6701-6705 (2019). 